\title{
Modified and conventional ultrafiltration during pediatric cardiac surgery: Clinical outcomes compared
}

Glyn D. Williams, MB, ${ }^{a, b}$ Chandra Ramamoorthy, MD, ${ }^{a, b}$ Larry Chu, MD, MS, ${ }^{a}$ Gregory B. Hammer, MD, ${ }^{a, b}$ Komal Kamra, MB, ${ }^{a, b}$ Madalane G. Boltz, MD, ${ }^{a}, b$ Krassimira Pentcheva, ${ }^{a}$ James P. McCarthy, CCP, and Vadiyala M. Reddy, MD ${ }^{\mathrm{a}, \mathrm{b}}$

From Stanford University School of Medicine $^{\mathrm{a}}$ and Lucile Packard Children's Hospital, ${ }^{\text {b }}$ Stanford, Calif.

Funding for this research was provided in part by Innovations In Patient Care Grant Program, Lucile Packard Children's Hospital, Stanford, Calif.

Received for publication Feb 8, 2006; revisions received May 3, 2006; accepted for publication May 12, 2006.

Address for reprints: Glyn David Williams, MB, Department of Anesthesia, Stanford University School of Medicine, 300 Pasteur Dr, H3587, Stanford, CA 94305-5640 (E-mail: jumbo@stanford.edu).

J Thorac Cardiovasc Surg 2006;132:1291-8

$0022-5223 / \$ 32.00$

Copyright () 2006 by The American Association for Thoracic Surgery

doi:10.1016/j.jtcvs.2006.05.059
Objective: This prospective study compared clinical outcomes after heart surgery between three groups of infants with congenital heart disease. One group received dilutional conventional ultrafiltration (group D), another received modified ultrafiltration (group $\mathrm{M}$ ), and a third group received both dilutional conventional and modified ultrafiltration (group B). We hypothesized that group B patients would have the best clinical outcome.

Methods: Children younger than 1 year undergoing heart surgery for biventricular repair by the same surgeon were randomly allocated to one of the three study groups. Patient management was standardized, and intensive care staff were blinded to group allocation. Primary outcome measure was duration of postoperative mechanical ventilation. Other outcome measures recorded included total blood products transfused, duration of chest tube in situ, chest tube output, and stays in intensive care and in the hospital.

Results: Sixty infants completed study protocol. Mean age and weight were as follows: group $\mathrm{D}(\mathrm{n}=19), 61$ days, $4.3 \mathrm{~kg}$; group $\mathrm{M}(\mathrm{n}=20), 64$ days, $4.5 \mathrm{~kg}$; and group $\mathrm{B}(\mathrm{n}=21), 86$ days, $4.4 \mathrm{~kg}$. Preoperative and intraoperative characteristics were similar between groups. Ultrafiltrate volumes obtained were $196 \pm 93 \mathrm{~mL} / \mathrm{kg}$ in group $\mathrm{D}, 105 \pm 33 \mathrm{~mL} / \mathrm{kg}$ in group $\mathrm{M}$, and $261 \pm 113 \mathrm{~mL} / \mathrm{kg}$ in group B. There were no significant differences between groups for any outcome variable. Technical difficulties prevented completion of modified ultrafiltration in 2 of 41 infants.

Conclusion: There was no clinical advantage in combining conventional and modified ultrafiltration. Because clinical outcomes were similar across groups, relative risks of the ultrafiltration strategies may influence choice.

$\mathrm{D}$ uring cardiopulmonary bypass (CPB) for cardiac surgery, children are subjected to anticoagulation, hemodilution, hypothermia, nonpulsatile blood flow, and exposure of blood to nonendothelialized surfaces. In response to these nonphysiologic conditions, patients initiate a systemic inflammatory response syndrome (SIRS) that increases total body water and may result in multi-organ dysfunction. SIRS is considered a major contributor to the increased morbidity and mortality associated with CPB in children. ${ }^{1,2}$

Ultrafiltration can ameliorate the effects of CPB by removing free water and some inflammatory mediators. ${ }^{3,4}$ Conventional ultrafiltration (CUF) is performed during CPB. ${ }^{5}$ If fluid is added to the CPB circuit during CUF to increase the volume of ultrafiltrate, the process is dilutional ultrafiltration (DCUF). Modified ultrafiltration (MUF) is conducted after CPB ${ }^{6}$ Ultrafiltration of the prime (PUF) before the onset of $\mathrm{CPB}$ is sometimes performed if the $\mathrm{CPB}$ circuit is primed with packed red 

Abbreviations and Acronyms
ANOVA $=$ analysis of variance
$\mathrm{CPB}=$ cardiopulmonary bypass
CUF = conventional ultrafiltration
DCUF = dilutional ultrafiltration
DHCA = deep hypothermic circulatory arrest
ICU = intensive care unit
MUF = modified ultrafiltration
PRBCs = packed red blood cells
PUF = ultrafiltration of prime before onset of $\mathrm{CPB}$
SIRS = systemic inflammatory response syndrome

blood cells (PRBCs). ${ }^{7}$ All these ultrafiltration methods for cardiac operations have been found clinically beneficial relative to unfiltered control $\mathrm{CPB}^{4-6,8-10}$ and can be used separately or combined in the same patient to provide potentially additive positive effects. ${ }^{11}$

Differences between DCUF and MUF merit consideration. Technically, DCUF demands little of the surgeon's attention, whereas MUF increases the complexity of the immediate postCPB period. DCUF enables removal of inflammatory mediators throughout $\mathrm{CPB}$ and does not prolong the duration of $\mathrm{CPB}$, but it can only achieve moderate hemoconcentration. MUF provides more effective hemoconcentration, ${ }^{12,13}$ but it extends the duration of patient exposure to nonendothelial surfaces ${ }^{14}$ and does not reduce plasma concentrations of inflammatory mediators in children. ${ }^{15}$ Both techniques are considered safe.

Controversy remains regarding the optimal ultrafiltration strategy. 5,8,13,16-21 Surrogate outcome measures, such as cytokine levels, have not been helpful in guiding the choice of ultrafiltration in children, because the relationships between plasma concentrations of proinflammatory and anti-inflammatory cytokines and patient outcome are poorly defined. ${ }^{12,13,15}$ Gaynor $^{12}$ stated, "Further studies are necessary to identify patients most likely to benefit from ultrafiltration, and to define standard protocols for use of ultrafiltration in infants and neonates undergoing CPB."

The aim of our prospective study was to compare clinical outcome after cardiac operations between three groups of infants. One group of patients received DCUF only, another group received MUF only, and the third group received both DCUF and MUF. On the premise that ultrafiltration is beneficial, the protocol was designed to optimize each ultrafiltration strategy. Study hypothesis was that the infants who underwent both DCUF and MUF would have a better clinical outcome than the other groups.

\section{Materials and Methods}

The study protocol was approved by the institutional review board, and informed parental consent was obtained before patient enrollment.

\section{Study Population}

Infants younger than 1 year were enrolled in this prospective, randomized study. With a random numbers table, patients were allocated to receive DCUF (group D), MUF (group M), or both DCUF and MUF (group B).

Inclusion criteria were gestational age greater than 37 weeks, postnatal age younger than 12 months, and scheduling for cardiac operations performed by V.M.R. Exclusion criteria were as follows: active noncardiac disease that was expected to compromise the patient's postoperative recovery; previous sternotomy, which may influence blood loss, an outcome variable; weight greater than $9 \mathrm{~kg}$, because of the need for a CPB oxygenator of greater flow capacity (to reduce CPB variables, the oxygenator was limited to one model); and single-ventricle palliation (surgeon's preference).

\section{Anesthesia, Surgery, and CPB}

Anesthesia was induced with sevoflurane or ketamine (1-2 mg/kg) and maintained with fentanyl (30-100 $\mu \mathrm{g} / \mathrm{kg})$, midazolam (0.1-0.4 $\mathrm{mg} / \mathrm{kg}$ ), and rocuronium bromide, supplemented with isoflurane. Preoperative steroids were not administered.

Anticoagulation was established with an initial bovine heparin dose of $400 \mathrm{U} / \mathrm{kg}$, and additional heparin was administered during CPB to maintain Celite-based activated clotting time greater than 480 seconds. The dose and adequacy of anticoagulation reversal by protamine were guided by heparin-protamine titration. Nonpulsatile CPB was performed with a hollow-fiber membrane oxygenator (Terumo Capiox RX05; Terumo Cardiovascular Systems, Ann Arbor, Mich) and nonocclusive roller pump. CPB circuit components, setup, and prime were standardized. The circuit was primed with normal saline solution, $25 \%$ albumin, mannitol, sodium bicarbonate, calcium chloride, methylprednisolone $(30 \mathrm{mg} / \mathrm{kg})$, and heparin. Banked PRBCs and fresh-frozen plasma were added to achieve a hematocrit of about $30 \%$ during initiation of $\mathrm{CPB}$. Hypothermia was induced in all patients, and blood gases were regulated according to the alpha-stat regimen unless deep hypothermia was required, in which case a $\mathrm{pH}$-stat regimen was used during cooling. Myocardial preservation was achieved with cold crystalloid cardioplegia. Transfusion therapy in the operating room and intensive care unit (ICU) was standardized to established protocols. Target post-CPB hematocrit varied from $35 \%$ to $50 \%$, depending on the patient's cardiac and respiratory status. Antifibrinolytics were not administered.

Whenever possible, the surgeon attempted to standardize the conduct of surgery, CPB, and ultrafiltration. Toward the end of $\mathrm{CPB}$, the usual time to initiate preparations for MUF, the perfusionist divulged the patient's study group allocation to the surgeon and anesthesiologist.

\section{Ultrafiltration}

The polysulfone hemofilter used (Minntech HPH 400; Minntech Corporation, Minneapolis, Minn) uses hollow-fiber technology and is rated to have a filtration cutoff to particles greater than 
TABLE 1. Demographics of the patient population

\begin{tabular}{lccc}
\hline Variable & Group D & Group M & Group B \\
\hline No. & 19 & 20 & 21 \\
Age (d, mean \pm SD) & $61.21 \pm 63.82$ & $64.30 \pm 73.89$ & $86.10 \pm 104.00$ \\
Weight (kg, mean \pm SD) & $4.27 \pm 1.29$ & $4.52 \pm 1.23$ & $4.35 \pm 1.51$ \\
Male sex (No.) & $9 / 19(47 \%)$ & $10 / 20(50 \%)$ & $13 / 21(62 \%)$ \\
\hline
\end{tabular}

$65,000 \mathrm{~d}$ molecular weight. A transmembrane pressure gradient of at least $200 \mathrm{~mm} \mathrm{Hg}$ was applied during ultrafiltration.

After the addition of blood products, hemofiltration of the $\mathrm{CPB}$ circuit prime was performed before $\mathrm{CPB}$ to adjust $\mathrm{pH}$ and electrolyte concentrations and to remove inflammatory mediators. ${ }^{22}$ Filtrate volume from PUF ranged from 100 to $200 \mathrm{~mL}$.

DCUF was performed throughout CPB to achieve a filtrate volume of at least $120 \mathrm{~mL} / \mathrm{kg}$. Fluids (crystalloid, PRBCs, or plasma) were added when necessary to provide sufficient volume in the CPB circuit to permit ultrafiltration.

Arteriovenous MUF was initiated after separation from CPB by standard technique. Blood from the aortic cannula and from the CPB circuit venous reservoir was pumped through the hemofilter and then warmed by a coiled heat exchanger (Medtronic MYOtherm XP cardioplegia delivery system; Medtronic Inc, Minneapolis, Minn) and returned through the cardioplegia circuit to the patient's venous cannulas. Infusion rates were adjusted to maintain appropriate central venous or left atrial pressure. MUF was terminated when red cell salvage of circuit contents was judged by the perfusionist to be complete.

\section{Postoperative Care}

The ICU staff participating in postoperative patient management were blinded to study group assignment. Goals for mechanical ventilator support depended on the patient's cardiorespiratory status. Weaning from ventilator support was initiated after the patient had exhibited clinical stability. When the child demonstrated the ability to sustain adequate spontaneous respiratory effort and required minimal supplemental oxygen, as assessed by arterial blood gas analysis, the child's trachea was extubated.

\section{Data Collection}

The primary patient outcome measure was duration of postoperative mechanical ventilation of the lungs. Secondary outcome measures were total volume and units of blood products transfused by weight of infant, duration of chest tube in situ, and ICU and hospital stays.

Data were recorded regarding patient demographics, preoperative clinical status, and the ultrafiltration methods used. Aspects of the intraoperative and postoperative courses were recorded for the first 5 days of ICU care or until discharge from the ICU, whichever was earlier. Details included transfusion therapy, cardiorespiratory function and support, fluid balance, nutrition, drug therapy, laboratory tests, perioperative adverse events, and the period from termination of $\mathrm{CPB}$ to end of surgery.

\section{Power Analysis}

With standard sample size calculation for a power of $0.80, P=$ .05 , equal variance and effect size similar to that reported for postoperative duration of lung ventilation, ${ }^{5}$ the sample size required was 51 ( $\mathrm{n}=17$ per group). Sample size calculations indicated that a sample of 51 would also be appropriate for the secondary outcome variables.

\section{Data Analyses}

Data were analyzed to ascertain whether groups D, M, and B were similar and could be compared. Clinical outcome was compared between all three groups of patients. Analysis of variance (ANOVA) was used to compare group means between the three types of ultrafiltration methods for continuous outcome measures. The Pearson $\chi^{2}$ was used to analyze categoric variables. Longitudinal continuous data was analyzed with mixed effects analysis. When data were not normally distributed, nonparametric statistical analyses (Kruskall-Wallis) were used. Computations were performed with SAS 9.1 (SAS Institute, Inc, Cary, NC).

\section{Results}

Sixty-two infants were enrolled in the study. Two subjects were excluded from data analysis for protocol violations. Of the remaining 60 patients, 19 received DCUF only (group D), 20 received MUF only (group $\mathrm{M}$ ) and 21 received both DCUF and MUF (group B).

Demographic characteristics of the three groups were similar and are presented in Table 1. Operations performed included repair of ventricular septal defect $(n=13)$, repair of tetralogy of Fallot $(n=12)$, repair of atrioventricular septal defect $(n=12)$, repair of transposition of the great arteries $(n=9)$, repair of total anomalous pulmonary venous return $(\mathrm{n}=3)$, complex repair of double-outlet right ventricle $(n=3)$, repair of multiple level obstruction of left heart $(n=2)$, repair of anomalous origin of coronary artery from pulmonary artery $(n=1)$, resection of cardiac tumor $(\mathrm{n}=1)$, repair of congenitally corrected transposition $(\mathrm{n}=$ $1)$, repair of cor triatriatum $(n=1)$, repair of truncus arteriosus $(\mathrm{n}=1)$, and repair of biventricular outflow obstruction $(\mathrm{n}=1)$. The operative procedure included valve repair in 5 cases (excluding tetralogy of Fallot and atrioventricular septal defect repairs) and aortic reconstruction in 14 cases. 
TABLE 2. Intraoperative characteristics of the patient population

\begin{tabular}{|c|c|c|c|c|}
\hline Characteristic & Group D & Group M & Group B & $P$ value \\
\hline Complex surgery (No.) & $7 / 19(37 \%)$ & $9 / 20(45 \%)$ & $9 / 21(43 \%)$ & .87 \\
\hline $\mathrm{CPB}$ prime $(\mathrm{mL}$, mean $\pm \mathrm{SD})$ & $459 \pm 43$ & $471 \pm 52$ & $476 \pm 47$ & .51 \\
\hline CPB duration (min, mean \pm SD) & $123 \pm 52$ & $142 \pm 57$ & $146 \pm 57$ & .40 \\
\hline Aortic crossclamp time (min, mean $\pm S D)$ & $94 \pm 104$ & $86 \pm 33$ & $86 \pm 44$ & .90 \\
\hline Minimum core temperature $\left({ }^{\circ} \mathrm{C}\right.$, mean $\left.\pm \mathrm{SD}\right)$ & $26.6 \pm 5.4$ & $25.1 \pm 5.3$ & $25.1 \pm 4.8$ & .56 \\
\hline Total heparin (units, mean \pm SD) & $5442 \pm 1111$ & $4935 \pm 1952$ & $5200 \pm 1072$ & .55 \\
\hline
\end{tabular}

$C P B$, cardiopulmonary bypass.

There were no significant differences in the prevalence of preoperative medication use or the need for preoperative mechanical ventilation. Study groups also did not differ significantly with respect to preoperative hematocrit, white blood cell count, electrolyte levels, and renal and coagulation laboratory test values. Selected intraoperative characteristics of patients are presented in Table 2. There were no significant differences between groups for duration of CPB, duration of crossclamping, minimum core temperature during $\mathrm{CPB}$, or total heparin dose. The distributions of individual anesthesiologists and perfusionists caring for these patients were not significantly different between groups. Groups did not differ significantly in the complexity of cardiac operations performed. None of the patients were subjected to deep hypothermic circulatory arrest (DHCA). Total volumes of ultrafiltrate obtained were $196 \pm 93$ $\mathrm{mL} / \mathrm{kg}$ (group D), $105 \pm 33 \mathrm{~mL} / \mathrm{kg}$ (group M), and $261 \pm$ $113 \mathrm{~mL} / \mathrm{kg}$ (group B).
MUF increased hematocrit values in group $\mathrm{M}$ from $39 \% \pm 6 \%$ to $47 \% \pm 6 \%(P<.0001)$ and in group B from $39 \% \pm 7 \%$ to $47 \% \pm 8 \%(P<.0001)$. In addition, MUF increased arterial blood pressures in group $\mathrm{M}$ from 57 to $84 \mathrm{~mm} \mathrm{Hg}$ systolic $(P<.0001)$ and from 34 to $51 \mathrm{~mm} \mathrm{Hg}$ diastolic $(P<.0001)$ and in group B from 58 to $76 \mathrm{~mm} \mathrm{Hg}$ systolic $(P<.0001)$ and from 35 to $48 \mathrm{~mm} \mathrm{Hg}$ diastolic $(P<.0001)$. Hematocrit and arterial blood pressure at equivalent time points did not change significantly for group D patients.

The primary and secondary outcome measures are shown in Table 3. There was no difference between groups in the duration of postoperative mechanical ventilation of the lungs, duration of chest tube placement, or ICU and hospital stays. Total blood product use, measured by volume per kilogram of body weight and by units per kilogram of body weight, also did not differ between groups. Blood products transfused during surgery and in the first 24 postoperative

TABLE 3. Principal outcome measures

\begin{tabular}{|c|c|c|c|c|}
\hline Outcome & Group D & Group M & Group B & $P$ value \\
\hline Duration of mechanical ventilation $(\mathrm{h})^{*}$ & & & & .57 \\
\hline $25 \%$ & 27.75 & 40.71 & 25.66 & \\
\hline $50 \%$ & 48.75 & 69.92 & 66.66 & \\
\hline $75 \%$ & 76.16 & 128.75 & 112.83 & \\
\hline Duration of chest tube in situ $(\mathrm{h})^{*}$ & & & & .20 \\
\hline $25 \%$ & 62.50 & 48.38 & 47.58 & \\
\hline $50 \%$ & 71.00 & 88.79 & 94.16 & \\
\hline $75 \%$ & 86.00 & 162.71 & 115.83 & \\
\hline Total volume of transfused blood products $(\mathrm{mL} / \mathrm{kg}) \dagger$ & $237.8 \pm 87.5$ & $223.9 \pm 91.0$ & $262.7 \pm 138$ & .54 \\
\hline Stay in ICU $(h)^{*}$ & & & & .82 \\
\hline $25 \%$ & 98.18 & 101.76 & 118.98 & \\
\hline $50 \%$ & 150.41 & 212.41 & 164.56 & \\
\hline $75 \%$ & 352.73 & 332.32 & 256.33 & \\
\hline Duration in hospital $(d)^{*}$ & & & & .49 \\
\hline $25 \%$ & 7 & 7 & 6 & \\
\hline $50 \%$ & 10 & 15 & 11 & \\
\hline $75 \%$ & 18 & 25 & 21 & \\
\hline
\end{tabular}

ICU, intensive care unit. *Data are presented as median and $25 \%$ and $75 \%$ quartiles, and Kruskal-Wallis test was computed. $\dagger$ Data are presented as mean $\pm S D$, and ANOVA was computed. 
hours were $1.87 \pm 0.79$ units $/ \mathrm{kg}$ (group D), $1.72 \pm 0.68$ units $/ \mathrm{kg}$ (group M), and $1.99 \pm 0.96$ units $/ \mathrm{kg}$ (group B) for all products combined and did not differ between groups $(P=.57$, ANOVA $)$. Likewise there were no differences in use (units per kilogram) of PRBCs $(P=.55)$, plasma $(P=$ $.39)$, platelets $(P=.65)$, or cryoprecipitate $(P=.76)$. Hematocrit values measured at 48 postoperative hours did not differ significantly between groups (mean \pm SD group D $44 \% \pm 5 \%$, group M $44 \% \pm 6 \%$, and group B $43 \% \pm$ $5 \%, P=.49$ ).

Additional clinical outcome parameters were then analyzed. There were no differences between groups for the following (data not presented): pulmonary compliance and resistance at 24 postoperative hours $(P=.55$ and $P=.10$, respectively, ANOVA); alveolar-arterial oxygen gradient at 24 postoperative hours ( $P=.71$, Kruskal-Wallis); systolic blood pressure preincision, at the end of operation, and at 24 postoperative hours $(P=.64, P=.41$, and $P=.69$, respectively, ANOVA), doses of inotropes used during the first 5 days in the ICU (dopamine $P=.11$, milrinone $P=$ .31 , random coefficient analysis); duration that a central venous catheter was in situ $(P=.24$, Kruskal-Wallis); interval between administration of protamine and termination of surgery ( $P=.71$, Kruskal-Wallis); chest tube output at 24 postoperative hours $(P=.77$, Kruskal-Wallis); and values of blood urea nitrogen and serum creatinine at 48 postoperative hours $(P=.38$ and $P=.30$, respectively, ANOVA).

Post hoc power analysis was based on a 2-sample $t$-test to resolve a 50\% change in group B with actual observed variance and assuming an $\alpha$ of 0.05 . The powers to resolve $50 \%$ change in outcome variables were $92 \%$ for duration of postoperative ventilation, $99 \%$ for duration of chest tube, and $99 \%$ for total volume of blood products transfused.

Of the 60 patients that completed study protocol, 2 died. Both were in study group $\mathrm{M}$ and were late deaths (postoperative days 29 and 95) that were unlikely to be related to the ultrafiltration technique used. Two patients (weight $3.9 \mathrm{~kg}$ and $1.8 \mathrm{~kg}$ ) allocated to group B were excluded from data analysis because the study protocol was not followed. In both cases, the patients had received DCUF but MUF was terminated prematurely because of systemic hypotension.

\section{Discussion}

We hypothesized that an ultrafiltration strategy that used DCUF to facilitate early modification of SIRS and MUF to maximize hemoconcentration would provide optimal outcome for infants undergoing cardiac operations. Our study found that the combination of MUF and DCUF afforded no additional benefit in terms of patient outcome relative to either MUF or DCUF alone. Technical complications were twice encountered during MUF.
Evidence has accumulated that ultrafiltration reduces postoperative morbidity after pediatric cardiac operations. Ultrafiltration has been shown to decrease total body water accumulation, decrease postoperative blood loss and blood product use, increase arterial blood pressure and improve left ventricular systolic function, improve the alveolar-arterial oxygen gradient and pulmonary compliance, decrease the frequency of pulmonary hypertensive episodes and the duration of postoperative ventilation, and decrease the incidence of pleural effusions after superior cavopulmonary connection and the Fontan procedure. ${ }^{12}$ Although the mechanisms by which ultrafiltration produces beneficial effects remain unclear, surmises include reduction of tissue edema, hemoconcentration, and removal of inflammatory mediators.

MUF was introduced in 1991 because CUF inadequately limited the postoperative accumulation of total body water in children. ${ }^{6}$ The introduction of zero balance ultrafiltration ${ }^{8}$ and DCUF ${ }^{5,23}$ permitted removal of large volumes of ultrafiltrate during $\mathrm{CBP}$ and prompted debate about the relative merits of MUF, DCUF, and a combination of the two techniques. ${ }^{6,8,16}$

Two recent reviews of ultrafiltration during cardiac operations concur that the results of published studies are conflicting and that further investigations are necessary to better define ultrafiltration strategies in the pediatric population. ${ }^{12,15}$ Pediatric studies that have compared ultrafiltration during CPB and MUF are listed in Table 4. A major problem that complicates interpretation of study findings is the lack of standardization in the performance of ultrafiltration. Factors that may influence study results include type of ultrafiltration during CPB (CUF, DCUF), type of MUF (arteriovenous, venovenous), duration of ultrafiltration during $\mathrm{CPB}$, volume of ultrafiltrate obtained, end point chosen for termination of MUF, the type of hemofilter, use of PUF, concomitant anti-inflammatory therapies (such as aprotinin or corticosteroids), patient characteristics (eg, young age, presence of pulmonary hypertension ${ }^{23}$ ), CPB variables (eg, prime volume and type), and complexity of cardiac surgery (eg, use of $\mathrm{DHCA}^{24}$ ). Additionally, several reports of ultrafiltration were retrospective or included historical control subjects.

Our single-institution, prospective, randomized study was designed to reduce confounding variables by blinding the ICU caregivers, standardizing intraoperative and postoperative care, and limiting the study population to infants. Also, it was hoped that by selecting this age group, detection of differences in outcome would be enhanced, because the benefits of ultrafiltration are more pronounced in infants undergoing complex cardiac operations. ${ }^{24}$ Twenty-nine of the study patients (48\%) were neonates.

Some of the beneficial effects of ultrafiltration may be transient and have minimal positive effect on clinical out- 
TABLE 4. Studies comparing MUF and conventional CUF in children undergoing cardiac surgery

\begin{tabular}{|c|c|c|c|c|c|c|}
\hline First author & Group & $\operatorname{Age}^{*}(\mathrm{mo})$ & n & Ultrafiltrate $(\mathrm{mL} / \mathrm{kg})$ & Hctt (\%) & Clinical outcome \\
\hline \multirow[t]{2}{*}{ Wang $^{18}$} & MUF & 62 & 24 & - & 18 & \multirow{2}{*}{$\begin{array}{l}\text { No difference in inotrope use, diuresis, duration of } \\
\text { ventilation, ICU stay }\end{array}$} \\
\hline & CUF & 44 & 26 & - & 18 & \\
\hline Thompson ${ }^{16}$ & CUF & 9 & 67 & 68 & $28-30$ & $\begin{array}{l}\text { No difference in blood product transfusions, } \\
\text { hemodynamics, left ventricle shortening, duration } \\
\text { of ventilation, ICU stay }\end{array}$ \\
\hline Maluf ${ }^{19}$ & MUF + CUF & 9 & 20 & 39 & 25 & $\begin{array}{l}\text { No difference in inotrope use, transfusions, duration } \\
\text { of ventilation, ICU stay, hospital stay }\end{array}$ \\
\hline Sever ${ }^{20}$ & CUF & 13 & 14 & $-\ddagger$ & $>20$ & $\begin{array}{l}\text { MUF + CUF: better hemodynamics, less bleeding } \\
\text { and transfusions, shorter duration of ventilation, } \\
\text { shorter ICU stay }\end{array}$ \\
\hline \multirow[t]{2}{*}{ Bando $^{5}$} & MUF + DCUF & 17 & 50 & 155 & $14-18$ & \multirow{2}{*}{$\begin{array}{l}\text { MUF + DCUF: high-risk patients§ had less } \\
\text { transfusions, better oxygenation, shorter duration } \\
\text { of ventilation, shorter ICU stay }\end{array}$} \\
\hline & CUF & 30 & 50 & 29 & $14-18$ & \\
\hline
\end{tabular}

MUF, modified ultrafiltration; CUF, conventional ultrafiltration; ICU, intensive care unit; DCUF, dilutional ultrafiltration. *Mean or median age of patients. $\dagger$ Target hematocrit during cardiopulmonary bypass. $\$$ Value not published. §High risk factors were neonatal age, pulmonary hypertension, and CBP duration longer than 120 minutes.

come. Keenan and colleagues ${ }^{25}$ reported that MUF, relative to nonultrafiltered control $\mathrm{CPB}$, resulted in a significant improvement in lung compliance immediately after CPB but not at the end of surgery or 24 postoperative hours. There were no differences between groups in duration of mechanical ventilation or ICU stay. With this in mind, we chose outcome variables that were of clinical relevance and likely to influence our future choice of ultrafiltration strat-1 egies (Table 3). None of these principal outcome measures differed significantly between groups. Other more subtle and perhaps transient outcome parameters were then compared between groups, but no significant differences were found. Parameters included postoperative measures of pulmonary function (compliance, resistance, alveolar-arterial oxygen gradient), cardiac function (arterial blood pressure, inotrope requirements, duration that central venous catheter was in situ), hemostasis (duration of intraoperative postCPB period, chest tube output), and renal function (blood urea nitrogen, creatinine).

Review of the publications listed in Table 4 suggests that differences in outcome between study groups are more likely when the groups being compared differ greatly in the volumes of ultrafiltrate obtained. This is not surprising, because the positive benefits of ultrafiltration correlate with the volume of filtrate removed. ${ }^{26}$ Total ultrafiltrate volumes for our study exceeded those reported in the studies summarized in Table 4. A recent publication ${ }^{27}$ that demonstrated efficacy of MUF in neonates obtained a mean ultrafiltrate volume of $104 \mathrm{~mL} / \mathrm{kg}$; our MUF ultrafiltrate volume was $105 \mathrm{~mL} / \mathrm{kg}$. The significant increases in hematocrit and arterial blood pressure during MUF provide additional evidence that MUF was adequately performed. ${ }^{3,26,27}$

For all our study patients, banked PRBCs were added to the CPB circuit prime and PUF was performed. PUF is reported to lower plasma concentrations of bradykinin and high-molecular weight kininogen and, relative to control procedures, result in less tissue edema, improved cardiorespiratory status, and reduced durations of mechanical ventilation and ICU stay. ${ }^{7,22,28}$ None of the studies listed in Table 4 ultrafiltered the heme prime before initiation of CPB. It is uncertain what influence PUF had on the outcome of our patients, but it may be that large ultrafiltrate volumes and early initiation of ultrafiltration are both important for improving clinical outcome.

There are other factors to consider. None of our study patients were submitted to DHCA; infants undergoing DHCA particularly benefited from MUF. ${ }^{24,27}$ With the exception of the study by Thompson and associates, ${ }^{16}$ the target hematocrit during CPB in our study was considerably higher than those of other studies (Table 4). Addition of methylprednisolone to the CPB prime may have modified the outcome of our patients. Unlike the other studies listed in Table 4, all our patients were infants and were operated on by the same surgeon.

Although ultrafiltration during and after CPB in children is considered safe, ${ }^{12}$ consideration of risk is warranted. ${ }^{25}$ Hemofiltration carries the potential for human and equipment error and increases plasma heparin concentration. 
Compared with DCUF, MUF has some additional risks. The aortic cannula may entrain air. Removal of blood from the systemic circulation may result in hemodynamic instability or impair aortopulmonary shunt flow. High flow rates through the ultrafilter decrease cerebral blood flow velocities and cerebral mixed venous oxygen saturation. ${ }^{29}$ In small infants, the aortic cannula may be obstructive, and its early removal may limit or prevent use of arteriovenous MUF. MUF extends the period of patient exposure to nonendothelialized surfaces. Cooling of the patient will occur if the ultrafiltered blood is inadequately warmed.

Two patients assigned to study group B did not receive MUF because of hemodynamic issues during the immediate post-CPB period. After assessing our data, we have opted not to provide MUF to infants undergoing cardiac operations, because MUF and DCUF were similarly beneficial but the incidence of complications, although uncommon, was greater for MUF than for DCUF. We combine PUF and DCUF.

Selection of ultrafiltration strategies appears linked to the conduct of CPB. Gaynor and coworkers ${ }^{27}$ retrospectively reviewed 99 neonates who underwent the first stage of Norwood reconstruction and noted that MUF was successfully and safely used in all cases. DHCA was universally used (mean duration 45 minutes), and the mean duration of CPB support was 100 minutes. In such instances, MUF seems a logical option because duration (and perhaps benefit) of DCUF would be limited if there is no blood flow for nearly half of the CPB period. At our institution, DHCA is seldom used for the first stage of Norwood reconstruction.

The conclusions of this study of infants may not be applicable to older children. MUF may be desirable for hemoconcentration in bigger children, in whom avoidance of exposure to donor blood products is feasible. Study weaknesses should be noted. It was impossible to completely blind intraoperative care providers to the method of ultrafiltration. Most of the outcome measures pertained to the patient's postoperative clinical course, however, and ICU caregivers were blinded. It is possible that the study had insufficient power to detect differences between groups, although post hoc power analysis indicates that this is unlikely.

In summary, this prospective, randomized study of 60 infants found no difference in clinical outcome between patients who received DCUF only, patients who received MUF only, and patients who received both DCUF and MUF.

\section{References}

1. Rubens FD, Mesana T. The inflammatory response to cardiopulmonary bypass: a therapeutic overview. Perfusion. 2004;19 Suppl 1:S5-12.

2. Seghaye MC, Grabitz RG, Duchateau J, Busse S, Dabritz S, Koch D, et al. Inflammatory reaction and capillary leak syndrome related to cardiopulmonary bypass in neonates undergoing cardiac operations. J Thorac Cardiovasc Surg. 1996;112:687-97.

3. Elliott MJ. Ultrafiltration and modified ultrafiltration in pediatric open heart operations. Ann Thorac Surg. 1993;56:1518-22.

4. Journois D, Pouard P, Greeley WJ, Mauriat P, Vouhe P, Safran D. Hemofiltration during cardiopulmonary bypass in pediatric cardiac surgery. Anesthesiology. 1994;81:1181-9.

5. Bando K, Turrentine MW, Vijay P, Sharp TG, Sekine Y, Lalone BJ, et al. Effect of modified ultrafiltration in high-risk patients undergoing operations for congenital heart disease. Ann Thorac Surg. 1998;66: 821-8.

6. Naik S, Knight A, Elliott MJ. A successful modification of ultrafiltration for cardiopulmonary bypass in children. Perfusion. 1991;6:41-50.

7. Shimpo H, Shimaoto A, Sawamura Y, Fujinaga K, Kanemitsu S. Ultrafiltration of the priming blood for cardiopulmonary bypass attenuates inflammatory response and improves postoperative clinical course in pediatric patients. Shock. 2001;16 Suppl 1:51-4.

8. Journois D, Israel-Biet D, Pouard P, Rolland B, Silvester W, Vouhe P, et al. High volume, zero-balanced hemofiltration to reduce delayed inflammatory response to cardiopulmonary bypass in children. Anesthesiology. 1996;85:965-76.

9. Davies MJ, Nguyen K, Gaynor JW, Elliott MJ. Modified ultrafiltration improves left ventricular systolic function in infants after cardiopulmonary bypass. J Thorac Cardiovasc Surg. 1998;115:361-70.

10. Hennein HA, Kiziltepe U, Barst S, Boccheiri KA, Hossain A, Call DR, et al. Venovenous modified ultrafiltration after cardiopulmonary bypass in children: a prospective randomized trial. J Thorac Cardiovasc Surg. 1999;117:496-505.

11. Ungerleider R. Effects of cardiopulmonary bypass and use of modified ultrafiltration. Ann Thorac Surg. 1998;65(6 Suppl):S35-9; discussion S39, S74-6.

12. Gaynor JW. The effect of modified ultrafiltration on the postoperative course in patients with congenital heart disease. Semin Thorac and Cardiovasc Surg Pediatr Card Surg Апnи. 2003;6:128-39.

13. Ramamoorthy C, Lynn A. Con: the use of modified ultrafiltration during pediatric cardiovascular surgery in not a benefit. $J$ Cardiothorac Vasc Anesth. 1998;12:483-5.

14. Booker PD, Davis AJ. Paediatric cardiac anaesthesia in the UKcurrent controversies. Anasthesiol Intensivmed Notfallmed Schmerzther. 2003;38:506-13.

15. Chew M. Does modified ultrafiltration reduce the systemic inflammatory response to cardiac surgery with cardiopulmonary bypass? Perfusion. 2004;19 Suppl 1:S57-60.

16. Thompson LD, McElhinney DB, Findlay P, Miller-Hance W, Chen MJ, Minami M, et al. A prospective randomized study comparing volume-standardized modified and conventional ultrafiltration in pediatric cardiac surgery. J Thorac Cardiovasc Surg. 2001;122:220-8.

17. Montenegro LM, Greeley WJ. Pro: the use of modified ultrafiltration during pediatric cardiac surgery is a benefit. $J$ Cardiothorac Vasc Anesth. 1998;12:480-2.

18. Wang M, Chiu I, Hsu C, Wang C, Lin P, Chang C, et al. Efficacy of ultrafiltration in removing inflammatory mediators during pediatric cardiac operations. Ann Thorac Surg. 1996;61:651-6.

19. Maluf MA, Mangia C, Silva, Carvalho WB, Carvalho AC, Buffolo E. Conventional and conventional plus modified ultrafiltration during cardiac surgery in high-risk congenital heart disease. J Cardiovasc Surg. 2001;42:465-73.

20. Sever K, Tansel T, Basaran M, Kafali E, Ugurlucan M, Ali Sayin O, et al. The benefits of continuous ultrafiltration in pediatric cardiac surgery. Scand Cardiovasc J. 2004;38:307-11.

21. Hiramatsu T, Imai Y, Kurosawa H, Takanashi Y, Aoki M, Shinoka $\mathrm{T}$, et al. Effects of dilutional and modified ultrafiltration in plasma endothelin-1 and pulmonary vascular resistance after the Fontan procedure. Ann Thorac Surg. 2002;73:861-5.

22. Nagashima M, Imai Y, Seo K, Terada M, Aoki M, Shinoka T, et al. Effect of hemofiltrated whole blood pump priming on hemodynamics and respiratory function after the arterial switch operation in neonates. Ann Thorac Surg. 2000;70:1901-6.

23. Bando K, Vijay P, Turrentine MW, Sharp TG, Means LJ, Ensing GJ, et al. Dilutional and modified ultrafiltration reduces pulmonary hyper- 
tension after operations for congenital heart disease: a prospective randomized study. J Thorac Cardiovasc Surg. 1998;115:517-25.

24. Naik SK, Knight A, Elliott M. A prospective randomized study of a modified technique of ultrafiltration during pediatric open-heart surgery. Circulation. 1991;84(5 Suppl):III4222-31.

25. Keenan HT, Thiagarajan R, Stephens KE, Williams G, Ramamoorthy C, Lupinetti FM. Pulmonary function after modified venovenous ultrafiltration in infants: a prospective, randomized trial. $J$ Thorac Cardiovasc Surg. 2000;119:501-5; discussion 506-7.

26. Gaynor JW. Use of ultrafiltration during and after cardiopulmonary bypass in children. $J$ Thorac Cardiovasc Surg. 2001;122:209-11.
27. Gaynor JW, Kuypers M, van Rossem M, Wernovsky G, Marino BS, Tabbutt S, et al. Hemodynamic changes during modified ultrafiltration immediately following the first stage of the Norwood reconstruction. Cardiol Young. 2005;15:4-7.

28. Sakuriai H, Maeda M, Sai N, Miyahara K, Nakayama M, Hasegawa H, et al. Extended use of hemofiltration and high perfusion flow rate in cardiopulmonary bypass improves perioperative fluid balance in neonates and infants. Ann Thorac Cardiovasc Surg. 1999;5:94-100.

29. Rodriguez RA, Ruel M, Broecker L, Cornel G. High flow rates during modified ultrafiltration decrease cerebral blood flow velocity and venous oxygen saturation in infants. Ann Thorac Surg. 2005;80:22-8. 